\title{
Interpretation of the energy spectrum observed with the Telescope Array surface detectors
}

\author{
E. Kido*1, O.E. Kalashev ${ }^{2}$ for the Telescope Array Collaboration ${ }^{\dagger}$ \\ ${ }^{1}$ Institute for Cosmic Ray Research, University of Tokyo \\ ${ }^{2}$ Institute for Nuclear Research of the Russian Academy of Sciences, Moscow, Russia \\ E-mail: ekidodicrr.u-tokyo.ac.ip \\ E-mail: kalashevdinr.ac.rud
}

We present the energy spectrum of ultra-high-energy cosmic rays (UHECRs) with energy E > $10^{18.2} \mathrm{eV}$ observed for five years of data by the surface detectors of the Telescope Array experiment. The broken power law to the spectrum contains two break points, a flattening known as the "ankle" or "dip" at $\mathrm{E}=10^{18.70} \mathrm{eV}$, and a steepening at $\mathrm{E}=10^{19.75} \mathrm{eV}$. These spectral features are related to the distribution of cosmic-ray sources, their injection spectra, and energy loss processes during the propagation of UHECRs in inter-galactic space. In this talk, we consider a phenomenological model of proton sources distributed either uniformly or following the largescale structure distribution. We show that in both cases it is possible to produce satisfactory fits assuming a power law injection spectrum and strong evolution of the source density with redshift. We also discuss constraints on the source model parameters, e.g. the injection power law and cosmological evolution.

The 34th International Cosmic Ray Conference,

30 July- 6 August, 2015

The Hague, The Netherlands

\footnotetext{
*Speaker.

${ }^{\dagger}$ Full author list: http://www.telescopearray.org/images/papers/ICRC2015-authorlist.pdf
} 


\section{Introduction}

The Telescope Array (TA) [U] is the largest cosmic-ray observatory in the Northern Hemisphere. The TA experiment has 507 surface particle detectors (SDs) on a square grid with $1.2 \mathrm{~km}$ spacing covering approximately $700 \mathrm{~km}^{2}$. The SDs are surrounded by three fluorescence detector (FD) stations (12, 12 and 14 telescopes). The duty cycle of the SD array is greater than $95 \%$ throughout 5-year observation period, whereas the FD duty cycle is about $10 \%$ because the data are taken only on moonless clear nights. The measurement of the energy spectrum using the TA SD data for four years was published in Ref. [2]. The energy spectrum was updated using the TA SD data for the five years between 2008 May 11 and 2013 May 4 [B]]. This energy spectrum shows the ankle at $10^{18.70} \mathrm{eV}$ and the cutoff at $10^{19.75} \mathrm{eV}$. An extended spectrum beyond the cutoff is ruled out with a statistical significance of $5.74 \sigma$. The suppression that is consistent with the GZK cutoff [ [⿴囗十, [] was first observed by the High Resolution Fly's Eye (HiRes) [可]. The Pierre Auger Observatory (PAO) also observed the flux suppresion above about $10^{19.5} \mathrm{eV}$ [四].

The interpretation of cosmic ray spectrum features crucially depends on assumptions about the UHECR composition. In particular the suppression of UHECR flux above $10^{19.75} \mathrm{eV}$ is expected for protons due to GZK mechanism [四, 回] while in the case of heavy nuclei as primaries this feature may signal the natural cutoff in the maximal acceleration energy of sources.

The measurement of the depth of shower maximum $\left(X_{\max }\right)$ in the events with energies above $10^{18.2} \mathrm{eV}$ by the TA FD in hybrid mode [ [8] as well as the previous measurements by HiRes [Q] observed light, largely protonic composition. However, Pierre Auger Observatory measurements suggest the presence of some light nuclei such as helium nuclei in the composition of cosmic rays at the highest energies [0]].

In this paper, we attempt to fit the energy spectrum measured by the TA SD with the pure proton source model. As it was pointed out in Ref. [W] this model applied to HiRes data is capable of explaining both ankle and cut-off features of the spectrum as a result of $e^{+} e^{-}$-pair production and pion production on the cosmic microwave background (CMB) without introducing any extra components or fine tuning the maximal source acceleration energy.

In the next section, the model calculations and the fitting procedure are explained, and the fitted results are shown in section [3. The systematic uncertainties of the conclusive fit parameters are described in section 4 , and the conclusions are shown in section 5 .

\section{Methodology}

The factors which define the observed cosmic ray spectrum can be divided in two groups:

- properties of sources i.e. distribution of sources in power, spectrum and maximal energy

- properties of the media i.e. photon backgrounds, magnetic fields and their evolution

Like in many previous works in this paper we attempt to fit the experimental data using simple phenomenological model for the source spectrum and evolution:

$$
Q(E, z)=\alpha E^{-p}(1+z)^{3+m}, E<E_{\max }, z<z_{\max },
$$


where $\alpha, p, m, E_{\max }$ and $z_{\max }$ are free parameters. The parameter $m$ parameterizes the evolution of the source density per comoving volume. $m=0$ if the source density is constant per comoving volume. The effect of sources located at $z>0.7$ is negligible for cosmic ray energies $E>10^{18.2}$ $\mathrm{eV}$. The parameter $E_{\max }$ in practice has small effect on fit goodness if $E_{\max }$ is greater than about $2 \times 10^{20} \mathrm{eV}$. Throughout this paper we use fixed values of $E_{\max }=10^{21} \mathrm{eV}$ and $z_{\max }=2$ unless explicitly stated. We also separately consider the special case when sources are distributed as local large scale structure (LSS) for which we use the distribution of galaxies from the Two Micron All Sky Survey (2MASS). We discuss the difference of the fit results with the assumption of source distribution of LSS in section 4.2 .

The main attenuation mechanism for UHECR protons is photopion production and $e^{+} e^{-}$-pair production on the cosmic microwave background (CMB). However interactions with infrared (and optic) background component (IRB) and deflections by the intergalactic magnetic field (IGMF) could lead to extra attenuation. The last two factors are currently poorly known. The existing model calculations of the IRB spectral density [12], [1], [4] may differ by factor of 2 at redshift $z=0$ and moreover the uncertainty grows with redshift.

The IGMF strength is even less constrained. Current theoretical and observational constraints on the IGMF are summarized in the review [L5]. The IGMF strength $B$ is stronger than about $10^{-17} \mathrm{G}$ and weaker than about $10^{-9} \mathrm{G}$ from the constraints. As it is shown in Ref. [एర]] the effect of magnetic fields on the propagation of protons with energies above $10^{18} \mathrm{eV}$ can be neglected if average distance between sources is less than about $50 \mathrm{Mpc}$ and IGMF strength is less than about $10^{-10} \mathrm{G}$. While the former condition is natural to assume for UHECR sources the latter is not necessary true. Nevertheless to limit the number of free model parameters below we consider the case of small IGMF $B<10^{-10} \mathrm{G}$ and fit the energy spectrum by proton sources above $10^{18.2} \mathrm{eV}$.

Finally to take into account systematic uncertainty in the measurement of cosmic ray energies we introduce shift of experimental energy scale as additional free parameter $\Delta \log E=\log E-$ $\log E_{\mathrm{obs}}$. Here and below $E_{\mathrm{obs}}$ is the measured energy and the base of the logarithm is 10 .

\subsection{Simulation of the propagation of protons}

We use publicly available numerical code [ㅁ] to simulate the attenuation of protons. This code was developed in Ref. [1]8]. The code calculates the average propagated energy spectrum by solving 1D transport equation assuming rectilinear propagation of cosmic rays. As mentioned above this assumption suggests that IGMF strength $B$ is less than about $10^{-10} \mathrm{G}$. The above code takes into account $e^{+} e^{-}$pair production process using continuous energy loss approximation, which is very precise for frequent interactions with small energy loss in a single interaction. For the pion production the code utilizes the interaction rates derived with SOPHIA code [एT]. The code contains several implementations of IRB models. Unless otherwise stated below we use the Kneiske et. al. best-fit model [ㅈ] for IRB. We discuss the uncertainties related to IRB model choice in section 4.3. We also employ CRPropa v2.0.3 propagation code [20] to estimate the systematic uncertainties of spectrum fitting.

\subsection{Model fitting of the data}

We convert the simulated energy flux to mean expected number of events in each energy bin using TA SD exposure and evaluated the consistency between the simulation and the data 
using $\chi^{2}$ values. We conservatively estimate the total flux determination uncertainties as $\sigma_{\mathrm{TOT}}=$ $\sqrt{\sigma_{\text {STAT }}^{2}+\sigma_{\text {SYS }}^{2}}$, where $\sqrt{N_{i}}$ is the statistical error of the observed number of events $N_{i}$ in each energy bin and $\sigma_{\mathrm{SYS}}$ is systematic uncertainty which is about $3 \%$ of the flux and mostly comes from the event reconstructions. We determine the confidence region of each free parameter using $\Delta \chi^{2}=$ $\chi^{2}-\chi_{\min }^{2}$, where $\chi_{\min }^{2}$ is the minimum value of $\chi^{2}$. We assumed that each free parameter follows a Gaussian distribution and that the true value of each free parameter is the best fit parameter. With these assumptions, $\Delta \chi^{2}$ corresponding to the coverage probability of the confidence region are given in Table 38.2 in Ref. [2]]. $\Delta \chi^{2} \leq 2.3$ condition is used to determine $68 \%$ confidence region within which 2 parameters simultaneously take on values. MINUIT package [22] is used for the $\chi^{2}$ minimization and the error analysis.

\section{Fit results}

We show the expected energy spectrum with source parameters determined by fitting the TA

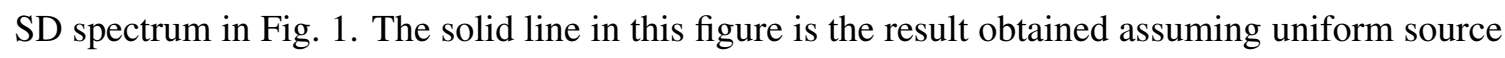
distribution. The best fit parameters are $p=2.21, m=6.7$ and $\Delta \log E=-0.03$. Systematic uncertainty of the energy determination is estimated to be $22 \%$ [3] , and the best fit shift of the energy scale $\Delta \log E$ is less than this. The best fit $\chi^{2} /$ d.o.f. $=12.4 / 17$ is obtained, so this model spectrum agrees with the data very well. The determined fit parameters $p, m$, and $\Delta \log E$ with statistical errors are $p=2.21_{-0.15}^{+0.10}, m=6.7_{-1.4}^{+1.7}, \Delta \log E=-0.03_{-0.03}^{+0.03}$. The correlation coefficient between $p$ and $m$ is -0.97 , the correlation coefficient between $p$ and $\Delta \log E$ is -0.35 and the correlation coefficient between $m$ and $\Delta \log E$ is 0.53 . The confidence region of values on which $p$ and $m$ simultaneously take is shown in Fig. \.

\section{Systematic uncertainties}

In this section we discuss theoretical modeling uncertainties.

\subsection{Dependence of the UHECR propagation code}

We have repeated the fitting procedure using the Monte Carlo code CRPropa ver.2.0.3 with modifications described in Ref. [ए]] to simulate propagation of UHECR. We get the difference in the parameter predictions $\Delta p=0.01, \Delta m=0.2$ and $\Delta(\Delta \log E)=0.01$. This difference is small compared to the parameters' confidence regions.

\subsection{LSS source distribution}

To model the UHECR sources distributed as the large scale structure, we use approximately 110,000 galaxy samples of 2MASS Galaxy Redshift Catalog (XSCz) that is derived from the 2MASS Extended Source Catalog (XSC) [R4]. The redshifts of most of the galaxies of XSCz catalog are obtained using the spectroscopy and the redshifts of some galaxies of this catalog are obtained using the photometry of 2MASS. We select the galaxies in the XSCz catalog with comoving distances smaller than $250 \mathrm{Mpc}$ and apparent magnitudes brighter than 12.5 in the $K_{s}$ band $(2.2$ $\mu \mathrm{m})$ and assume a uniform matter distribution beyond $250 \mathrm{Mpc}$. The galaxies within $5 \mathrm{Mpc}$ are excluded because their number is too small to represent statistical sample of LSS. We introduce 


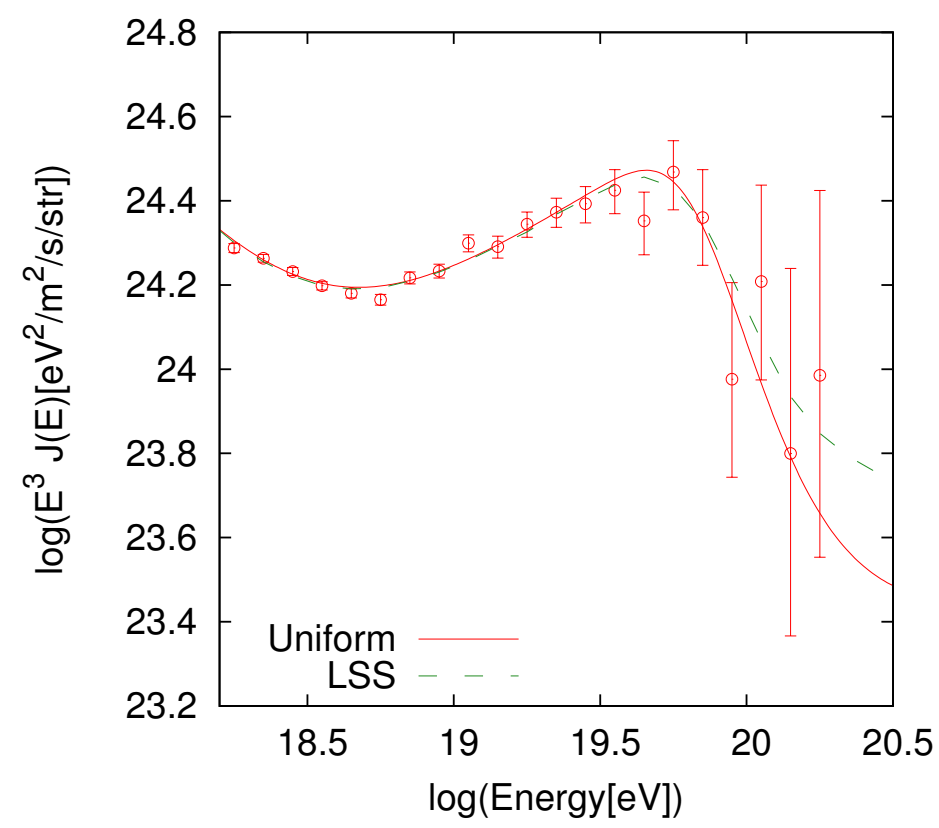

Figure 1: The red data points denote the energy spectrum measured by TA SD. The red solid line denotes the best-fit expected energy spectrum with $p=2.21, m=6.7, \Delta \log E=-0.03$ for a uniform distribution of UHECR sources. $\chi^{2} /$ d.o.f. is $12.4 / 17$. In this figure, the energy scale of the data points is fixed and the energy scale of the model is shifted by $\Delta \log E=+0.03$. The green dashed line denotes the best-fit expected energy spectrum when UHECRs are distributed along the LSS.

a weighting factor for each selected galaxy to take into account faint galaxies below the limit of apparent magnitude 12.5 following the procedure described in [25]]. First we calculate the dependence of $\sum_{i} w_{i} A_{i}$ on distance from the galaxies, where $w_{i}$ is the weight of each galaxy and $A_{i}$ is the relative TA SD exposure in the direction of the galaxy. Then we simulate energy spectra from the source distribution $\sum_{i} w_{i} A_{i}$ using the modified CRPropa ver.2.0.3 and assuming the injection spectrum (…1). Figure 3 shows the dependence of $\sum_{i} w_{i}$ on the comoving distance from the galaxies. Some bumps can be seen in this figure and this feature reflects the concentration of local matter density. The bump around $20 \mathrm{Mpc}$ shows the feature of the Virgo cluster for example.

We obtain the best fit parameters by repeating the procedure described in section $\square$. The expected flux with these parameters is shown as a dashed green line in Fig. $\mathbf{W}$. The differences of the best fit parameters between LSS and uniform source distribution are $\Delta p=0.02, \Delta m=0.3$ and $\Delta(\Delta \log E)=0.02$.

\subsection{Dependence on the IRB}

To estimate uncertainty related to poor knowledge of IRB we have repeated the spectrum fitting procedure using IRB models of Ref. [ए2], [ए3] and [प4]]. The largest difference in terms of best fit parameters was obtained between the "best fit" model of Ref. [[13] and "minimal" model of Ref. [प]]]: $\Delta p=0.03, \Delta m=0.3$ and $\Delta(\Delta \log E)=0.01$. 


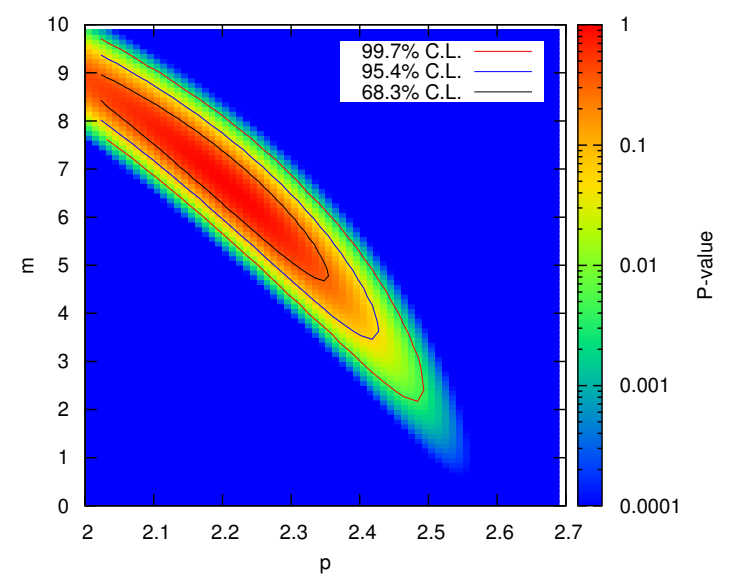

Figure 2: The confidence regions of values on which fitting parameters $p$ and $m$ simultaneously take are plotted with a color map for uniform source distribution. The systematic flux uncertainties related to the event reconstructions are taken into account. Other fitting parameters $\alpha$ and $\Delta \log E$ are determined to minimize $\chi^{2}$ for each $p$ and $m$ in this color map. The $x$-axis is the injection power index $p$ of sources. The $y$-axis is $m$ of the number evolution $(1+z)^{m}$ of comoving source density.

\section{Conclusions}

We fitted a simple phenomenological model (ㅁ] assuming pure proton injection to the TA SD spectrum above $10^{18.2} \mathrm{eV}$. We obtained $\chi^{2} /$ d.o.f. $=12.4 / 17$ with reasonable best fit energy scale $\Delta \log E=-0.03$, and other parameters $p=2.21_{-0.15}^{+0.10}$ (stat. + syst.) and $m=6.7_{-1.4}^{+1.7}$ (stat. + syst.). We also consider the effect of sources being distributed inhomogeneously (following LSS distribution) and uncertainties related to usage of different propagation codes and IRB models. We conclude that the largest uncertainty is related to the choice of the IRB model: $\Delta p=0.04, \Delta m=0.3$.

\section{Acknowledgments}

The Telescope Array experiment is supported by the Japan Society for the Promotion of Science through Grants-in-Aid for Scientific Research on Specially Promoted Research (21000002) "Extreme Phenomena in the Universe Explored by Highest Energy Cosmic Rays" and for Scientific Research (19104006), and the Inter-University Research Program of the Institute for Cosmic Ray Research; by the U.S. National Science Foundation awards PHY-0307098, PHY-0601915, PHY-0649681, PHY-0703893, PHY-0758342, PHY-0848320, PHY-1069280, PHY-1069286, PHY1404495 and PHY-1404502; by the National Research Foundation of Korea (2007-0093860, R3210130, 2012R1A1A2008381, 2013004883); by the Russian Academy of Sciences, RFBR grants 11-02-01528a and 13-02-01311a (INR), IISN project No. 4.4502.13, and Belgian Science Policy under IUAP VII/37 (ULB). The foundations of Dr. Ezekiel R. and Edna Wattis Dumke, Willard L. Eccles, and George S. and Dolores Doré Eccles all helped with generous donations. The State of Utah supported the project through its Economic Development Board, and the University of Utah 


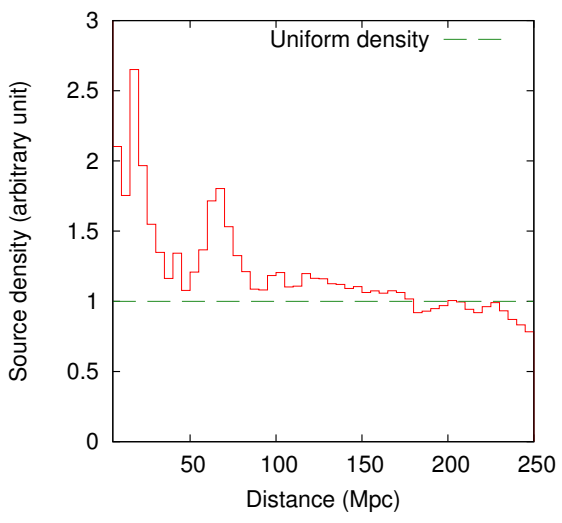

Figure 3: Calculated $\sum_{i} w_{i}$ distribution of galaxies in the $\mathrm{XSCz}$ catalogue, where $w_{i}$ is the weight of $i$-th galaxy. In this figure, calculated $\sum_{i} w_{i}$ within a slice of $5 \mathrm{Mpc}$ divided by squared comoving distance is presented. The x-axis is comoving distance. The uniform density is calculated dividing total $\sum_{i} w_{i}$ inside of $250 \mathrm{Mpc}$ by the comoving volume of the sphere with $250 \mathrm{Mpc}$ radius. This uniform density is used beyond $250 \mathrm{Mpc}$ in the model of the LSS source distribution.

through the Office of the Vice President for Research. The experimental site became available through the cooperation of the Utah School and Institutional Trust Lands Administration (SITLA), U.S. Bureau of Land Management, and the U.S. Air Force. We also wish to thank the people and the officials of Millard County, Utah for their steadfast and warm support. We gratefully acknowledge the contributions from the technical staffs of our home institutions. An allocation of computer time from the Center for High Performance Computing at the University of Utah is gratefully acknowledged.

\section{References}

[1] http://www.telescopearray.org/

[2] T. Abu-Zayyad et al., ApJ. 768, L1 (2013).

[3] D. Bergman et al. (the Telescope Array Collaboration), 33rd ICRC (Rio de Janeiro), CR-EX, Id:221 (2013).

[4] K. Greisen, Phys. Rev. Lett. 16, 748 (1966).

[5] G.T. Zatsepin and V.A. Kuz'min, J. Exp. Theor. Phys. Lett. 4, 78 (1966).

[6] R.U. Abbasi et al. (the HiRes Collaboration), Phys. Lett. B619, 271 (2005).

[7] J. Abraham et al. (the Pierre Auger Collaboration), Phys. Lett. B685, 239 (2010).

[8] R.U. Abbasi et al. (the Telescope Array Collaboration), Astropart. Phys. 64, 49 (2015) [arXiv:1407.1726v3 [astro-ph.HE]].

[9] R.U. Abbasi et al. (the HiRes Collaboration), Phys. Rev. Lett. 104, 161101 (2010).

[10] A. Aab et al. (the Pierre Auger Collaboration), Phys. Rev. D. 90, 122006 (2014).

[11] V.S. Berezinsky, A. Gazizov, \& S. Grigorieva, Phys. Rev. D 74, 043005 (2006). 
[12] A. Franceschini, G. Rodighiero and M. Vaccari, Astron. Astrophys. 487, 837 (2008) [arXiv:0805.1841 [astro-ph]]. F. W. Stecker, M. A. Malkan and S. T. Scully, Astrophys. J. 761, 128 (2012). S. T. Scully, M. A. Malkan and F. W. Stecker, [arXiv:1401.4435 [astro-ph.HE]]. J. R. Primack, R. C. Gilmore and R. S. Somerville, AIP Conf. Proc. 1085, 71 (2009); J. D. Finke, S. Razzaque and C. D. Dermer, Astrophys. J. 712, 238 (2010). Y. Inoue, S. Inoue, M. A. R. Kobayashi, R. Makiya, Y. Niino and T. Totani, Astrophys. J. 768, 197 (2013) [arXiv:1212.1683 [astro-ph.CO]].

[13] T. M. Kneiske et al., Astron. Astrophys. 386, 1 (2002); ibid., 413, 807 (2004).

[14] T. M. Kneiske and H. Dole, [arXiv:1001.2132 [astro-ph.CO]].

[15] R. Durrer and A. Neronov, Astron. Astrophys. Rev. 21, 62 (2013) [arXiv:1303.7121 [astro-ph.CO]].

[16] V. Berezinsky and A. Z. Gazizov, Astrophys. J. 669, 684 (2007) [arXiv:0702102 [astro-ph]].

[17] O. E. Kalashev and E. Kido, JETP 120, 5 (2015) [arXiv:1406.0735 [astro-ph.HE]].

[18] O.E. Kalashev, PhD thesis, INR RAS, Moscow 2003. G.B. Gelmini, O. Kalashev and D. V. Semikoz, JCAP 1201, 044 (2012) [arXiv:1107.1672 [astro-ph.CO]]. K. Arisaka, G.B. Gelmini, M.D. Healy, O. E. Kalashev and J. Lee, JCAP 0712, 002 (2007) [arXiv:0709.3390 [astro-ph]].

[19] A. Mücke, R. Engel, J. P. Rachen, R.J. Protheroe, T. Stanev, Computer Physics Communications 124, 290 (2000).

[20] K. -H. Kampert, J. Kulbartz, L. Maccione, N. Nierstenhoefer, P. Schiffer, G. Sigl and A. R. van Vliet, Astropart. Phys. 42, 41 (2013) [arXiv:1206.3132 [astro-ph.IM]].

[21] K.A. Olive et al. (Particle Data Group) Chin. Phys. C 38, 090001 (2014).

[22] F. James and M. Roos, Comput. Phys. Commun. 10, 343 (1975).

[23] D. Ivanov, PhD thesis, Rutgers, The State University of New Jersey 2012.

[24] T. Jarrett, Publ. Astron. Soc. Austral. 21, 396 (2004) [arXiv:0405069 [astro-ph]].

[25] T. Abu-Zayyad et al. ApJ. 757, 26 (2012).

[26] G. Gelmini, O. E. Kalashev and D. V. Semikoz, Astropart. Phys. 28, 390 (2007) [arXiv:0702464 [astro-ph]].

[27] M. Ackermann et al., ApJ. 799, 86 (2015).

[28] V. Berezinsky and O.E. Kalashev in preparation.

[29] M.G. Aartsen et al., Phys. Rev. D 88, 063002 (2013).

[30] G.W. Feldman and R.D. Cousins, Phys. Rev. D 57, 3873 (1998). 